Two-qubit entanglement dynamics for two different non-Markovian environments

This article has been downloaded from IOPscience. Please scroll down to see the full text article.

2010 Phys. Scr. 2010014014

(http://iopscience.iop.org/1402-4896/2010/T140/014014)

View the table of contents for this issue, or go to the journal homepage for more

Download details:

IP Address: 147.163.21.168

The article was downloaded on 01/10/2010 at 08:57

Please note that terms and conditions apply. 


\title{
Two-qubit entanglement dynamics for two different non-Markovian environments
}

\author{
Bruno Bellomo $^{1}$, Rosario Lo Franco ${ }^{1}$, Sabrina Maniscalco ${ }^{2}$ \\ and Giuseppe Compagno ${ }^{1}$ \\ ${ }^{1}$ CNISM and Dipartimento di Scienze Fisiche ed Astronomiche, Università di Palermo, via Archirafi 36, \\ 90123 Palermo, Italy \\ ${ }^{2}$ Department of Physics and Astronomy, University of Turku, Turun yliopisto, FIN-20014 Turku, Finland \\ E-mail: lofranco@ fisica.unipa.it
}

Received 29 March 2010

Accepted for publication 17 June 2010

Published 30 September 2010

Online at stacks.iop.org/PhysScr/T140/014014

\begin{abstract}
We study the time behaviour of entanglement between two noninteracting qubits, each immersed in its own environment for two different non-Markovian conditions: a high- $Q$ cavity slightly off-resonant with the qubit transition frequency and a nonperfect photonic band gap (PBG). We find that revivals and retardation of entanglement loss may occur by adjusting the cavity-qubit detuning, in the first case, while partial entanglement trapping occurs in a nonideal PBG.
\end{abstract}

PACS numbers: 03.67.-a, 03.67.Mn, 03.65.Yz, 03.65.Ud

(Some figures in this article are in colour only in the electronic version.)

\section{Introduction}

Real quantum systems unavoidably interact with their surroundings, undergoing a consequent decoherence and entanglement loss [1]. It is known that two entangled qubits embedded in Markovian (memoryless) environments may become completely disentangled at a finite time, in spite of an exponential decay of the single-qubit coherence [2, 3]. This entanglement sudden death (ESD or early-stage disentanglement), which has been experimentally revealed $[4,5]$, places a serious limit on the storage times of entanglement for practical purposes, e.g., for the realization of quantum memory banks [6]. A realistic quantum computer will probably have to take into account this quantum dynamical drawback. It is therefore important to study the possible physical conditions where entanglement can be maintained.

Entanglement losses during the evolution crucially depend on the particular noise acting on the system. Under Markovian-noise conditions the quantum process is typically irreversible. Differently structured environments or strong coupling can give rise to non-Markovian noise (environment with memory) whose effects on the entanglement dynamics are currently being subjected to investigation [6]. In this context, the cases of two noninteracting qubits embedded either in separated high- $Q$ cavities $[7,8]$ or in a common cavity [9] supporting a mode resonant with the qubit transition frequency have been analysed, showing that revivals of the initial two-qubit entanglement can occur. When the qubits share a common environment, it has also been shown that entanglement can be preserved by means of the quantum Zeno effect [10]. Moreover, entanglement trapping is achievable when two independent qubits are embedded in an ideal photonic band gap (PBG) material (photonic crystal) [11, 12].

The aim of this paper is therefore to deepen the analysis of entanglement evolution in non-Markovian environments, considering in particular two different effective spectral conditions of the environment-qubit system simulating, respectively, a cavity with a mode slightly off-resonant with the qubit transition frequency and a nonperfect PBG at the qubit transition frequency. In this paper, we highlight the differences with previous studies and we discuss the optimal physical parameters for observing entanglement revivals and for preservation of entanglement.

\section{Model}

We consider a system composed of two parts $\tilde{S}=\tilde{A}, \tilde{B}$, each one consisting of a two-level system (qubit) $S=A, B$ interacting with a reservoir $R_{S}=R_{A}, R_{\mathrm{B}}$. 
The single part $\tilde{S}$ 'qubit $S+\operatorname{reservoir} R_{S}$ ' is described by the Hamiltonian

$$
\hat{H}_{\tilde{S}}=\hbar \omega_{0} \hat{\sigma}_{+} \hat{\sigma}_{-}+\sum_{k} \hbar\left[\omega_{k} \hat{b}_{k}^{\dagger} \hat{b}_{k}+\left(g_{k} \hat{\sigma}_{+} \hat{b}_{k}+g_{k}^{*} \hat{\sigma}_{-} \hat{b}_{k}^{\dagger}\right)\right],
$$

where $\omega_{0}$ is the transition frequency, $\sigma \pm$ are the qubit raising and lowering operators, $b_{k}^{\dagger}, b_{k}$ are the creation and annihilation operators and $g_{k}$ is the coupling constant of mode $k$ with frequency $\omega_{k}$. When the environment is at zero temperature, the single-qubit reduced density matrix $\hat{\rho}^{S}(t)$ can be written, in the basis $\{|1\rangle,|0\rangle\}$, as [1]

$$
\hat{\rho}^{S}(t)=\left(\begin{array}{cc}
\rho_{11}^{S}(0)|q(t)|^{2} & \rho_{10}^{S}(0) q(t) \\
\rho_{01}^{S}(0) q^{*}(t) & \rho_{00}^{S}(0)+\rho_{11}^{S}(0)\left(1-|q(t)|^{2}\right)
\end{array}\right) .
$$

From the equation above, it is readily seen that the single-qubit dynamics depends only on the function $q(t)$ that in turn is determined by the reservoir spectral density. Indeed, $q(t)$ obeys the differential equation $\dot{q}(t)=-\int_{0}^{t} \mathrm{~d} t_{1} f(t-$ $\left.t_{1}\right) q\left(t_{1}\right)$, and the correlation function $f\left(t-t_{1}\right)$ is related to the spectral density $J(\omega)$ of the reservoir by $f(t-$ $\left.t_{1}\right)=\int \mathrm{d} \omega J(\omega) \exp \left[\mathrm{i}\left(\omega_{0}-\omega\right)\left(t-t_{1}\right)\right]$. The solution of the associated algebraic equation for $\dot{q}(t)$, obtained through Laplace transforms, is $\bar{q}(s)=q(0) /[s+\bar{f}(s)]$, where $\bar{q}(s)$ and $\bar{f}(s)$ are the Laplace transforms of $q(t)$ and $f\left(t-t_{1}\right)$.

A crucial quantity for our study is the explicit analytic expression of the two-qubit reduced density matrix at time $t$. For the system of noninteracting qubits in separated environments considered here, this can be obtained by a procedure based on the knowledge of the single-qubit dynamics [7]. In fact, given the time-dependent single-qubit density matrix elements as $\rho_{i i^{\prime}}^{A}(t)=\sum_{l l^{\prime}} A_{i i^{\prime}}^{l l^{\prime}}(t) \rho_{l l^{\prime \prime}}^{A}(0), \quad \rho_{j j^{\prime}}^{B}(t)=\sum_{m m^{\prime}} B_{j j^{\prime}}^{m m^{\prime}}(t) \rho_{m m^{\prime}}^{B}(0)$, the time-dependent two-qubit density matrix elements are [7]

$$
\rho_{i i^{\prime}, j j^{\prime}}(t)=\sum_{l l^{\prime}, m m^{\prime}} A_{i i^{\prime}}^{l l^{\prime}}(t) B_{j j^{\prime}}^{m m^{\prime}}(t) \rho_{l l^{\prime}, m m^{\prime}}(0),
$$

where $i, j, l, m=0,1$. The two-qubit density matrix $\hat{\rho}(t)$ is thus obtained by means of equation (3) for an arbitrary two-qubit initial condition, its elements depending only on their initial values and on the function $q(t)$ [7, 8]. In the following, these density matrix elements will be meant in the standard computational basis $\mathcal{B}=\{|1\rangle \equiv|11\rangle,|2\rangle \equiv$ $|10\rangle,|3\rangle \equiv|01\rangle,|4\rangle \equiv|00\rangle\}$.

\subsection{Initial states}

With regard to the initial state, we will limit our analysis to the case of initial pure Bell-like states

$$
|\Phi\rangle=\alpha|01\rangle+\beta \mathrm{e}^{\mathrm{i} \delta}|10\rangle, \quad|\Psi\rangle=\alpha|00\rangle+\beta \mathrm{e}^{\mathrm{i} \delta}|11\rangle,
$$

with $\alpha, \beta$ real and $\alpha^{2}+\beta^{2}=1$. For $\alpha= \pm \beta=1 / \sqrt{2}$, these states coincide with the Bell states. Bell-like states have the property that their resulting density matrix has an X structure (only diagonal and antidiagonal density matrix elements different from zero). Under our dynamical conditions, the $\mathrm{X}$ structure is maintained during the two-qubit evolution, so that the two-qubit density matrix at time $t$ will also have an $\mathrm{X}$ structure.

\subsection{Concurrence}

In order to quantify the entanglement during the evolution of the bipartite system, we use the concurrence $C$ [13]. The concurrence at time $t$ for an initial general $\mathrm{X}$ state can be easily computed by exploiting the fact that the $\mathrm{X}$ structure is preserved here and by using equations (2) and (3). The expression of the concurrence is given by [14]

$$
\begin{aligned}
C_{\rho}^{\mathrm{X}}(t)= & 2 \max \left\{0, K_{1}(t), K_{2}(t)\right\} \\
K_{1}(t)= & |q(t)|^{2}\left\{\rho_{23}(0)-\sqrt{\rho_{11}(0)}\left[\rho_{44}(0)+\rho_{11}(0)\right.\right. \\
& \left.\left.\times\left(1-|q(t)|^{2}\right)^{2}+\left(\rho_{22}(0)+\rho_{33}(0)\right)\left(1-|q(t)|^{2}\right)\right]^{\frac{1}{2}}\right\} \\
K_{2}(t)= & |q(t)|^{2}\left[\rho_{14}(0)-\sqrt{\rho_{22}(0)+\rho_{11}(0)\left(1-|q(t)|^{2}\right)}\right. \\
& \left.\times \sqrt{\rho_{33}(0)+\rho_{11}(0)\left(1-|q(t)|^{2}\right)}\right]
\end{aligned}
$$

These formulas are quite general since their form does not explicitly depend on the particular choice of the environment, but only on the Hamiltonian model of equation (1) and on the chosen initial state. The explicit time dependence of concurrence depends on the explicit form of the function $q(t)$ and thus it contains information about the environment structure. In the following, we shall consider two different environment structures with given spectral densities, which in turn determine the explicit form of $q(t)$.

\section{Spectral density effect on entanglement dynamics}

We shall now analyse the evolution of concurrence for two different spectral densities: a single Lorentzian simulating a cavity with a mode nonresonant with the qubit transition frequency and a nonperfect PBG at the qubit transition frequency.

\subsection{Off-resonant high-Q cavity}

As a first example we take the spectral distribution $J(\omega)$ of the electromagnetic field inside a high- $Q$ cavity supporting a mode detuned by $\Delta$ from the qubit (two-level atom) transition frequency $\omega_{0}$, resulting from the combination of the environment spectrum and the system-environment coupling. It has the Lorentzian form [1]

$$
J(\omega)=\frac{1}{2 \pi} \frac{\Gamma \lambda^{2}}{\left(\omega_{0}-\Delta-\omega\right)^{2}+\lambda^{2}},
$$

where $\Gamma$ is the qubit free-space linewidth and $\lambda$ the spectral width of the coupling. The parameter $\lambda$ is then connected to the reservoir correlation time $\tau_{\mathrm{B}}$ by the relationship $\tau_{\mathrm{B}} \approx \lambda^{-1}$. The relaxation timescale $\tau_{\mathrm{R}}$ over which the state of the system changes is related to $\Gamma$ by $\tau_{\mathrm{R}} \approx \Gamma^{-1}$. The correlation function corresponding to this $J(\omega)$ is $f\left(t-t_{1}\right)=$ $\frac{\Gamma \lambda}{2} \exp \left[-(\lambda-\mathrm{i} \Delta)\left(t-t_{1}\right)\right]$. Using this correlation function, the Laplace transform of $q(t)$ is

$$
\bar{q}(s)=1 /\left[s+\frac{1}{2} \frac{\Gamma \lambda}{s-(\lambda-\mathrm{i} \Delta)}\right]
$$




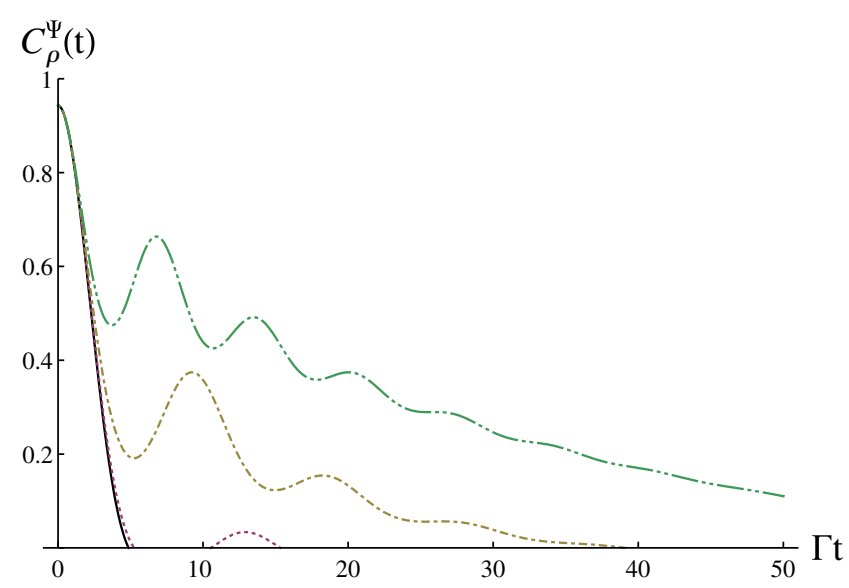

Figure 1. Nonresonant cavity: $\lambda=0.1 \Gamma$. Concurrence as a function of dimensionless quantities $\Gamma t$ starting from the initial state

$\hat{\rho}^{\Psi}(0)=|\Psi\rangle\langle\Psi|$ with $\alpha=1 / \sqrt{3}$ for different values of detuning $\Delta$ : $\Delta=0$ (solid curve), $\Delta=2 \lambda$ (dotted curve), $\Delta=5 \lambda$

(long-short-dashed curve), $\Delta=8 \lambda$ (long-short-short-dashed curve).

and the inverse Laplace transform finally gives

$$
q(t)=\mathrm{e}^{-((\lambda-\mathrm{i} \Delta) / 2) t}\left[\cosh \left(\frac{d t}{2}\right)+\frac{\lambda-\mathrm{i} \Delta}{d} \sinh \left(\frac{d t}{2}\right)\right],
$$

where $d=\sqrt{(\lambda-\mathrm{i} \Delta)^{2}-2 \Gamma \lambda}$.

In the resonant limit, $\Delta=0$, the correlation function has an exponential form with $\lambda$ the decay rate. In the analysis of the function $q(t)$ of equation (7) with $\Delta=0$, a weak $(\Gamma<\lambda / 2)$ and a strong $(\Gamma>\lambda / 2)$ coupling regime can be distinguished $[1,15]$. In the weak coupling regime, the relaxation time is greater than the reservoir correlation time $\left(\tau_{\mathrm{R}}>2 \tau_{\mathrm{B}}\right)$ and the behaviour of $q(t)$ is essentially a Markovian exponential decay controlled by $\Gamma$. In the strong coupling regime, the reservoir correlation time is greater than, or of the same order as, the relaxation time $\left(\tau_{\mathrm{R}}<2 \tau_{\mathrm{B}}\right)$ and non-Markovian effects become relevant. Within this regime, $q(t)$ presents oscillations describing a quasi-coherent exchange of energy between the qubit and the reservoir.

3.1.1. Entanglement dynamics. We now investigate the entanglement dynamics of the two-qubit system considering in detail the effect of the detuning $\Delta$, by using the concurrence obtained by equation (5) with $q(t)$ given by equation (7). The evolution of concurrence for various values of the detuning is shown in figure 1 when the cavity bandwidth $\lambda$ is smaller than the free-space atomic linewidth $\Gamma(\lambda=0.1 \Gamma)$ and the initial state is not maximally entangled, in particular $\hat{\rho}^{\Psi}(0)=$ $|\Psi\rangle\langle\Psi|$ with $\alpha=1 / \sqrt{3}$. In the resonant case, it is found, as is known, that the state suffers ESD [7]. However, from the plot one observes that on increasing the detuning, the entanglement decay slows down. In particular, for $\Delta=2 \lambda$ we also have revivals of entanglement after a finite period of time when the two qubits are not entangled. The phenomena of the slowing down of entanglement decay and of the entanglement revivals are a clear manifestation of the environment memory effects.

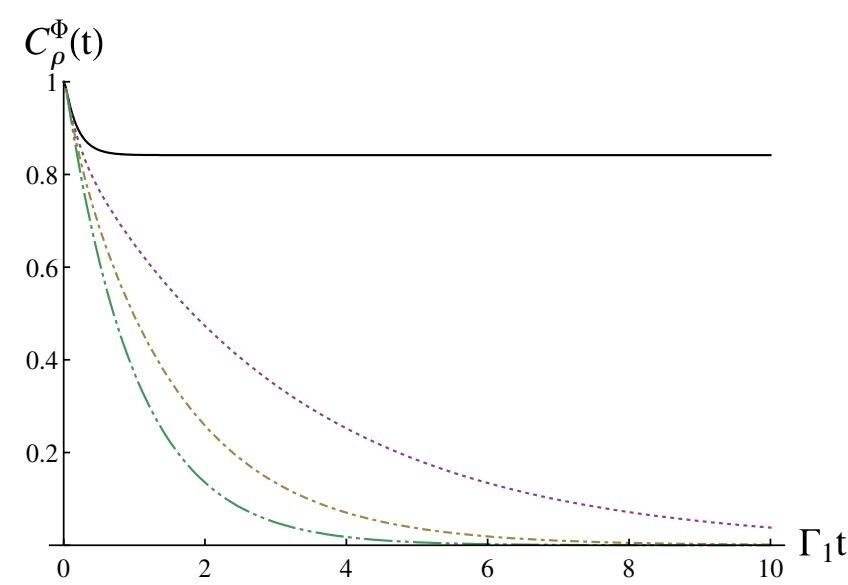

Figure 2. Nonperfect PBG case: $\lambda_{1}=10 \lambda_{2}=50 \Gamma_{1}$. Concurrence as a function of the dimensionless quantity $\Gamma_{1} t$ starting from the initial state $\hat{\rho}^{\Phi}(0)=|\Phi\rangle\langle\Phi|$ with $\alpha=\beta=1 / \sqrt{2}$ for different values of $\Gamma_{2}: \Gamma_{2}=\Gamma_{1}$ (solid curve), $\Gamma_{2}=\Gamma_{2} / 3$ (dotted curve), $\Gamma_{2}=2 \Gamma_{1} / 3$ (long-short-dashed curve), $\Gamma_{2}=0$ (long-short-short-dashed curve).

\subsection{Nonperfect $P B G$}

As a second example of the non-Markovian environment, we consider a spectral density of the form [16]

$$
J(\omega)=\frac{1}{2 \pi}\left(\frac{\Gamma_{1} \lambda_{1}^{2}}{\left(\omega-\omega_{0}\right)^{2}+\lambda_{1}^{2}}-\frac{\Gamma_{2} \lambda_{2}^{2}}{\left(\omega-\omega_{0}\right)^{2}+\lambda_{2}^{2}}\right),
$$

which represents a Lorentzian with a dip used as a model to simulate the spontaneous decay of a qubit in a nonperfect PBG. In equation (8), $\lambda_{1}$ represents the bandwidth of the flat background continuum, $\lambda_{2}$ the width of the gap, and $\Gamma_{1}$ and $\Gamma_{2}$ the strength of the background and the gap, respectively. The spectral density must be positive; this implies $\Gamma_{1} \lambda_{1}^{2}>\Gamma_{2} \lambda_{2}^{2}$ (condition for $J(\omega)$ to be positive at large $\omega$ ) and $\Gamma_{1}>\Gamma_{2}$ (condition for $J(\omega)$ to be positive at the centre of resonance). Combining these two relationships the condition $\Gamma_{1} \lambda_{1}>$ $\Gamma_{2} \lambda_{2}$ must be satisfied, this being also the condition for a localized dip [16]. In the case $\Gamma_{1}=\Gamma_{2}$ the spectral density reduces exactly to zero at the centre of the gap $\left(\omega=\omega_{0}\right)$, leading to population trapping [16]. For this form of $J(\omega)$, one obtains for the correlation function $f\left(t-t_{1}\right)=$ $\left(\Gamma_{1} \lambda_{1} \mathrm{e}^{-\lambda_{1}\left(t-t_{1}\right)}-\Gamma_{2} \lambda_{2} \mathrm{e}^{-\lambda_{2}\left(t-t_{1}\right)}\right) / 2$ and the Laplace transform of $q(t)$ becomes

$$
\bar{q}(s)=\frac{\left(\lambda_{1}+s\right)\left(\lambda_{2}+s\right)}{s^{3}+s^{2}\left(\lambda_{1}+\lambda_{2}\right)+s\left(\lambda_{1} \lambda_{2}+\Lambda\right)+\lambda_{1} \lambda_{2} \Gamma_{\mathrm{d}}},
$$

where $\Lambda=\left(\Gamma_{1} \lambda_{1}-\Gamma_{2} \lambda_{2}\right) / 2$ and $\Gamma_{d}=\left(\Gamma_{1}-\Gamma_{2}\right) / 2$. By inverting the Laplace transform, one finally obtains

$$
q(t)=\sum_{i} \frac{u_{i}^{2}+u_{i}\left(\lambda_{1}+\lambda_{2}\right)+\lambda_{1} \lambda_{2}}{\left(u_{i}-u_{j}\right)\left(u_{i}-u_{k}\right)} \mathrm{e}^{u_{i} t}
$$

where $i, j, k=1,2,3$ are all different indexes and $u_{i}$ are the three solutions of the third-degree equation appearing in the denominator of equation (9). The explicit expressions of $u_{i}$ are rather complex and we shall not report them here.

3.2.1. Entanglement dynamics. We are now ready to analyse the entanglement dynamics of the two-qubit system in a 
nonperfect $\mathrm{PBG}$ as described by the spectral density of equation (8), by using the concurrence of equation (5) with $q(t)$ given by equation (10). In figure 2 , we investigate the evolution of concurrence for $\lambda_{1}=10 \lambda_{2}=50 \Gamma_{1}$ and varying $\Gamma_{2}$. In order to obtain higher values of concurrence for long times, we consider an initial state different from that of the previous analysis (section 3.1.1), namely the Bell state $\hat{\rho}^{\Phi}(0)=|\Phi\rangle\langle\Phi|(\alpha=\beta=1 / \sqrt{2})$. For $\Gamma_{2}=\Gamma_{1}$, the spectral density goes to zero at the centre of the gap and, as a consequence, we obtain entanglement trapping (similarly to what happens in [11]). The other curves are obtained for decreasing values of $\Gamma_{2}: \Gamma_{2}=2 \Gamma_{2} / 3, \Gamma_{2}=\Gamma_{1} / 3$ and $\Gamma_{2}=0$. Smaller values of $\Gamma_{2}$ correspond to a smaller dip of the spectral density at $\omega=\omega_{0}$. In particular, for $\Gamma_{2}=0$, the shape of the spectral density is again a simple Lorentzian. In figure 2 the value of $\lambda_{1}$ is chosen so that we are in the weak coupling regime (see section 3.1) and a Markovian decay occurs. Finally, the plot shows how on decreasing $\Gamma_{2}$ the trapping of entanglement is lost and the entanglement decay speeds up even more.

\section{Conclusions}

In this paper, we have extended previous analysis on the entanglement dynamics of two noninteracting qubits embedded in bosonic environments at zero temperature. We have examined two different spectral densities corresponding to two different environments: the first case considered is a Lorentzian spectrum representing a high- $Q$ cavity out of resonance with the qubit transition frequency; the second case is a nonperfect PBG. The first case has allowed us to analyse the role of the cavity-qubit detuning comparing it to the known resonant case. In particular, as expected, an increase in the entanglement lifetime is observed when the detuning is increased. On the other hand, the second spectral density has permitted the study of entanglement dynamics when ideal conditions of the PBG (spectral density exactly equal to zero for a frequency equal to the qubit transition frequency) are not satisfied. In this case, it has been found that increasing the value of the spectral density in the central frequency (qubit transition frequency) entanglement trapping, which is expected for the ideal case, vanishes while entanglement decay speeds up. This study has thus enlarged the knowledge of entanglement evolution under different non-Markovian conditions, providing more hints for future investigations on this topic.

\section{Acknowledgments}

RLF (GC) acknowledges partial support by MIUR project II04C0E3F3 (II04C1AF4E) Collaborazioni Interuniversitarie ed Internazionali tipologia C. SM acknowledges financial support from the Turku Collegium of Science and Medicine, the Academy of Finland, the Väisälä Foundation, the Magnus Ehrnrooth Foundation and the Turku University Foundation.

\section{References}

[1] Breuer H P and Petruccione F 2002 The Theory of Open Quantum Systems (Oxford: Oxford University Press)

[2] Diósi L 2003 Lect. Notes Phys. 622157

[3] Yu T and Eberly J H 2004 Phys. Rev. Lett. 93140404

[4] Almeida M P et al 2007 Science 316579

[5] Laurat J, Choi K S, Deng H, Chou C W and Kimble H J 2007 Phys. Rev. Lett. 99180504

[6] Yu T and Eberly J H 2009 Science 323598

[7] Bellomo B, Lo Franco R and Compagno G 2007 Phys. Rev. Lett. 99160502

[8] Bellomo B, Lo Franco R and Compagno G 2008 Phys. Rev. A 77032342

[9] Mazzola L, Maniscalco S, Piilo J, Suominen K A and Garraway B M 2009 Phys. Rev. A 79042302

[10] Maniscalco S, Francica F, Zaffino R L, Gullo N L and Plastina F 2008 Phys. Rev. Lett. 100090503

[11] Bellomo B, Lo Franco R, Maniscalco S and Compagno G 2008 Phys. Rev. A 78060302

[12] Wang F Q, Zhang Z M and Liang R S 2008 Phys. Rev. A 78042320

[13] Wootters W K 1998 Phys. Rev. Lett. 802245

[14] Yu T and Eberly J H 2007 Quantum Inf. Comput. 7459

[15] Maniscalco S and Petruccione F 2006 Phys. Rev. A 7301211

[16] Garraway B M 1997 Phys. Rev. A 554636 